\title{
Mitochondrial Fission: Regulation and ER Connection
}

\author{
Hakjoo Lee, and Yisang Yoon*
}

\begin{abstract}
Fission and fusion of mitochondrial tubules are the main processes determining mitochondrial shape and size in cells. As more evidence is found for the involvement of mitochondrial morphology in human pathology, it is important to elucidate the mechanisms of mitochondrial fission and fusion. Mitochondrial morphology is highly sensitive to changing environmental conditions, indicating the involvement of cellular signaling pathways. In addition, the well-established structural connection between the endoplasmic reticulum (ER) and mitochondria has recently been found to play a role in mitochondrial fission. This minireview describes the latest advancements in understanding the regulatory mechanisms controlling mitochondrial morphology, as well as the ER-mediated structural maintenance of mitochondria, with a specific emphasis on mitochondrial fission.
\end{abstract}

\section{INTRODUCTION}

Mitochondria are eukaryotic organelles originating from ancient bacterial endosymbionts, which equipped host cells with a new, efficient means of energy production utilizing oxygen. In addition to the well-known bioenergetic role of mitochondria, their cell biological aspect has drawn a great deal of attention recently. It is thought that engulfment of bacteria through a phagocytic process during early endosymbiosis might have contributed to the unique double membrane structure of mitochondria. Overall cellular distribution and organization of mitochondria vary greatly in different organisms and tissues, presumably as an adaptative process that occurred when eukaryotic cells became multicellular organisms with highly differentiated organ systems, each having specific functions.

While their appearances are diverse in different tissues, mitochondria in many cell types are highly dynamic. All cellular membranous organelles have a dynamic nature, changing

Department of Physiology, Medical College of Georgia, Georgia Regents University, Augusta, GA 30912, USA

${ }^{*}$ Correspondence: yyoon@gru.edu

Received 7 November, 2013; accepted 10 November, 2013; published online 19 February, 2014

Keywords: dynamin, endoplasmic reticulum, ER-mitochondria contact, mitochondria, mitochondrial dynamics shape and distribution to some degree and communicating structurally and functionally through membrane trafficking. However, unlike the behavior of other cellular organelles, mitochondrial dynamics are much more robust and easily observable. They frequently divide and fuse, and rapidly move to different locations, displaying a widely varying range of shapes and locations in a relatively short time frame. These highly dynamic characteristics of mitochondria are suggested to be associated with their bioenergetic activity, as metabolic and environmental stresses profoundly influence mitochondrial dynamics. Although mitochondrial fission and fusion have been studied for some time now, the detailed connection between mitochondrial dynamics and bioenergetic activity is still not fully understood. Recently, several cellular signaling mechanisms that are evoked in different metabolic and environmental conditions were found to regulate mitochondrial dynamics. In this short review, we will discuss recent developments in understanding the regulatory mechanisms of mitochondrial morphology with a specific focus on mitochondrial fission and the role of ER.

\section{MITOCHONDRIAL FISSION AND FUSION BY DRPS}

Mitochondria in cells take many shapes, ranging from small vesicles to large blobs or elongated tubules depending on cell types. Mitochondria in most cultured mammalian cells are organized in reticular networks along the cytoskeletal system. Individual mitochondria undergo frequent fission and fusion, which occur in balanced frequency to maintain consistent overall cellular mitochondrial organization. The main proteins mediating fission and fusion of mitochondrial tubules are dynaminrelated proteins (DRPs). Mitochondrial DRPs include dynaminrelated/like protein 1 (Drp1/DLP1), Mitofusin (Mfn), and OPA1 (optic atrophy 1). Drp1/DLP1 mediates mitochondrial fission whereas Mfn and OPA1 mediate fusion of outer and inner membranes, respectively. DRPs are evolutionarily conserved. Most notably in budding yeast, Dnm1 is a DLP1 homologue, whereas Fzo1 and Mgm1 are homologues of Mfn and OPA1, respectively.

Conventional dynamin has been shown to function as a membrane pinching protein at the neck of a vesicle budding from the plasma membrane as well as other intracellular organelles through its GTPase activity (Hinshaw, 2000; McNiven et al., 2000). The mitochondrial DRPs mediate fission and fusion, presumably through similar membrane remodeling activity. The mitochondrial fission dynamin DLP1 assembles into a homooligomeric macromolecular complex with a ring-like structure 
(Smirnova et al., 2001; Yoon et al., 2001). Mixing DLP1 with liposomes induces helical assembly of DLP1 onto the liposome surface to form long lipid tubules, suggesting its membrane pinching activity (Yoon et al., 2001). More recent in vitro studies of purified yeast Dnm1 using cryoelectron microscopic analyses revealed a GTP hydrolysis-mediated membrane remodeling activity of this protein. This study showed that the diameter of Dnm1-decorated tubules were significantly decreased by two fold upon GTP hydrolysis, which was accompanied by rapid dissociation of Dnm1 from the constricted lipid tubule (Mears et al., 2011). These structural analyses suggest that Dnm1 exerts a contractile force on the membrane to mediate mitochondrial fission. Recently, it has been shown that endoplasmic reticulum (ER) tubules wrap around and constrict mitochondrial tubules (Friedman et al., 2011), providing sites at the mitochondrial tubule to accommodate the Dnm1 ring structure. The role of the ER in mitochondrial fission and ER-mitochondria contacts will be further discussed in the later part of this article.

In additon to DLP1/Dnm1, the inner membrane fusion dynamin OPA1 has been shown to tubulate liposomes in vitro (Ban et al., 2010). However, the mechanism by which this membrane-tubulating activity of OPA1 contributes to inner membrane fusion is not yet known. Considering that fusion requires mixing of lipid molecules between fusion partners, it is possible that OPA1 induces the protrusion of a slender lipid tubule at the tip of the fusing membrane, allowing close contact and subsequent breakage of the fusing bilayers. Lipid remixing and the formation of a new bilayer would form fusion pore that expands to complete fusion. While outer membrane fusion may involve a similar activity, no membrane tubulation activity has been identified for Mfn yet.

\section{PROTEINS WORKING WITH DLP1 IN MITOCHONDRIAL FISSION}

DLP1 is a cytosolic protein that needs to be recruited to mitochondria for fission to occur. Unlike conventional dynamin, DLP1 lacks the lipid-binding pleckstrin homology domain, suggesting that interaction with mitochondrial resident proteins is at play to assist fission (Mears et al., 2011). In yeast, Fis1 is the mitochondrial outer membrane protein, acting as a receptor for the Dnm1-Mdv1/Caf4 complex. Mdv1 and a similar molecule, Caf4, are the molecular adaptor protein that links Fis1 and Dnm1 at the mitochondrial surface (Griffin et al., 2005; Tieu et al., 2002). The mitochondrial fission process appears evolutionarily divergent, as Mdv1/Caf4-like protein is not present in mammals. Instead, additional mitochondrial proteins have been found to act as DLP1 receptors in mammals. Fis1, mitochondrial fission factor (Mff), mitochondrial dynamics protein of $49 \mathrm{kDa}$ and 51 $\mathrm{kDa}$ (MiD49/MiD51), and mitochondrial elongation factor 1 (MIEF1, identical to MiD51) have all been suggested to function as DLP1 receptors (Otera et al., 2010; Palmer et al., 2011; 2013; Zhao et al., 2011). Fis1 appears to distribute throughout the outer membrane, whereas Mff and MiDs are punctate on mitochondrial tubules and exhibit a stronger interaction with DLP1 than with Fis1. A recent study using null cell lines for Fis1 and/or Mff indicates that Fis1 and Mff contribute to DLP1 localization and oligomerization at the mitochondria independently of each other, with Mff having a more predominant role (Loson et al., 2013). In addition, MiD49 and MiD51 appear to mediate DLP1 recruitment in the absence of Mff and Fis1 (Loson et al., 2013). Several cellular signaling mechanisms have been found to regulate DLP1 recruitment and mitochondrial fission.

\section{REGULATION OF MITOCHONDRIAL FISSION}

Multiple mechanisms have been identified that regulate DLP1 to alter mitochondrial fission. These include transcriptional regulation, proteasomal degradation, reversible phosphorylation, sumoylation, S-nitrosylation, and O-glycosylation (Cho et al., 2009; Gawlowski et al., 2012; Guo et al., 2013; Horn et al., 2011; Nakamura et al., 2006; Wasiak et al., 2007; Yonashiro et al., 2006). Among these, reversible phosphorylation of DLP1 has been most extensively studied. DLP1 has two known phosphorylation sites that regulate its activities (Fig. 1). Both phosphorylation sites are located at the C-terminal domain, with the 2 sites located 20 amino acids apart. The upstream phosphorylation site $\left(\right.$ Ser $\left._{C D K 1}\right)$ is phosphorylated by CDK1 and ERK1/2, and PKC $\delta$ (Qi et al., 2011; Taguchi et al., 2007; Yu et al., 2011), whereas the downstream site (Ser PKA ) is phosphorylated by cyclic AMP-dependent protein kinase (PKA), calcium/calmodulin-dependent protein kinase l $\alpha(\mathrm{CaMKl} \alpha)$ and Rho-associated coiled coil-containing protein kinase 1 (ROCK1) (Chang and Blackstone, 2007; Han et al., 2008; Wang et al., 2012a).

Reversible phosphorylation of DLP1

PKA-phosphorylated DLP1 shows decreased GTPase activity (Chang and Blackstone, 2007) and localizes to the cytosol, resulting in elongated mitochondria due to decreased fission (Fig. 1). Starvation, stress, exercise, and $\beta$-adrenergic activation have been shown to increase PKA-mediated DLP1 phosphorylation (Cribbs and Strack, 2007; Gomes et al., 2011). In both neurons and cardiomyocytes, A kinase anchoring protein 1 (AKAP1, also known as AKAP121) at the mitochondrial surface targets PKA to the outer mitochondrial membrane and enhances DLP1 phosphorylation to inhibit mitochondrial fission (Kim et al., 2011; Merrill et al., 2011). Under hypoxic conditions, however, the ubiquitin ligase Siah2 ubiquitinates AKAP1 for degradation, decreasing DLP1 phosphorylation and thus increasing fission (Kim et al., 2011). In a parallel mechanism, AKAP1 also binds to Fis1 to prevent DLP1 recruitment. Hypoxic degradation of AKAP1 allows the DLP1-Fis1 interaction, resulting in enhanced mitochondrial fission (Kim et al., 2011).

PKA-phosphorylated DLP1 is dephosphorylated by the $\mathrm{Ca}^{2+}$ dependent phosphatase calcineurin (Cribbs and Strack, 2007). Mitochondrial depolarization caused by FCCP or arachidonic acid increases cytosolic $\mathrm{Ca}^{2+}$ concentration to activate calcineurin. The dephosphorylated DLP1 then localizes to mitochondria and fragments mitochondria (Cereghetti et al., 2008) (Fig. 1). Neuronal death following oxygen-glucose deprivation depends on the DLP1-calcineurin interaction through the calcineurindocking motif found in DLP1 (Slupe et al., 2013). Calcineurin protein level is also under tight regulation by miR-499, a microRNA that is abundant in cardiac tissue. Increasing miR-499 decreases calcineurin level and increases DLP1 phosphorylation, inducing mitochondrial elongation and protecting the cardiac tissue from ischemia-reperfusion injury (Wang et al., 2011). In addition to calcineurin, protein phosphatase 2A (PP2A) has also been implicated as a phosphatase for PKA-phosphorylated DLP1 in neurons (Fig. 1). The process of DLP1 phosphorylation and dephosphorylation by PKA and PP2A in developing neurons has been shown to regulate dendritogenesis and synaptogenesis, respectively, by altering mitochondrial $\mathrm{Ca}^{2+}$ buffering capacity (Dickey and Strack, 2011). Furthermore, the mitochondrial phosphoglycerate mutase/protein phosphatase PGAM5 has been shown to dephosphorylate the phosphoSer $_{P K A}$ of DLP1, resulting in mitochondrial fragmentation during necrosis that is induced by TNF- $\alpha$, reactive oxygen species 


$\begin{array}{lllllllll}0 & 100 & 200 & 300 & 400 & 500 & 600 & 700 & 800 \mathrm{AA}\end{array}$

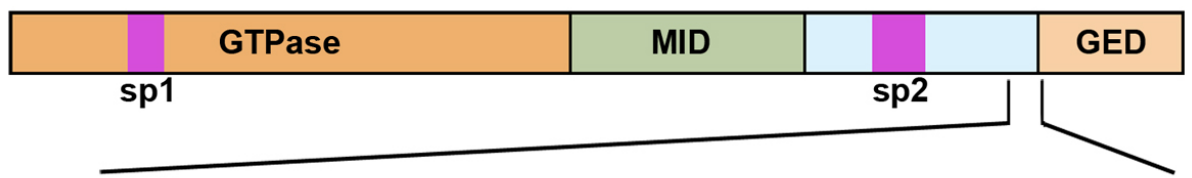

PIMPASPQKGHAVNLLDVPVPVARKLSAREQRD
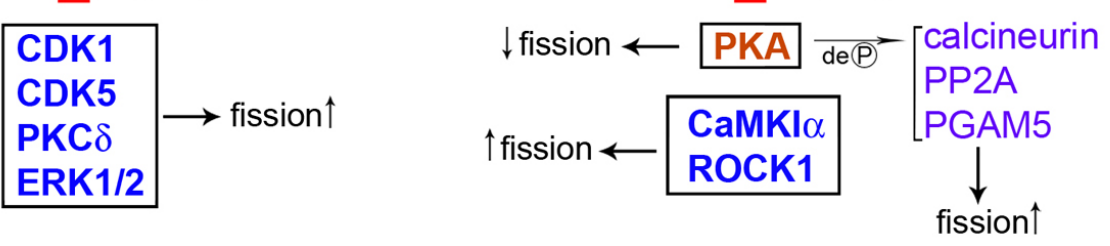

Fig. 1. Two serine residues in the DLP1 molecule that regulate mitochondrial fission. The C-terminal amino acid sequence of DLP1 contains two serine residues that undergo phosphorylation. Two purple boxes, sp1 and sp2, are regions of alternative splicing that produces different sizes of spliced variants. Kinases that phosphorylate DLP1 and increase fission are in blue color. DLP1 phosphorylation by PKA decreases fission. Dephosphorylation of PKA-phosphorylated DLP1 increases fission.

(ROS), and calcium ionophore (Wang et al., 2012b) (Fig. 1).

CaMK1 $\alpha$ has been shown to phosphorylate DLP1 at the same site $\left(\mathrm{Ser}_{\mathrm{PKA}}\right)$ upon $\mathrm{Ca}^{2+}$ influx through voltage-dependent calcium channels in neurons (Han et al., 2008) (Fig. 1). Additionally, ROCK1 also directly interacts with and phosphorylates DLP1 at Ser PKA $_{1}$ under high glucose conditions in kidney podocytes and endothelial cells (Wang et al., 2012a). Interestingly, despite phosphorylating the same site, CaMKl $\alpha$ - and ROCK1mediated phosphorylations result in enhanced fission, the opposite effect of PKA-phosphorylation (Fig. 1).

The other phosphorylation in DLP1

The upstream serine residue Ser CDK1 $_{1}$ of DLP1 is phosphorylated by the mitosis-promoting factor CDK1/cyclin B (Taguchi et al., 2007) (Fig. 1). CDK1-phosphorylated DLP1 induces mitochondrial fragmentation at the onset of mitosis to facilitate even distribution of mitochondria to daughter cells. DLP1 phosphorylation by CDK1 is initiated by the mitotic kinase Aurora A. Aurora $A$ activates the small GTPase RALA, which in turn translocates to mitochondria along with the RALA effector RALBP1 and DLP1. RALBP1 also brings CDK1/cyclin B to DLP1 for phosphorylation (Kashatus et al., 2011). Instead of dephosphorylation, DLP1 undergoes degradation through a cell cycle E3 ubiquitin ligase, APC/C (anaphase-promoting complex/cyclosome), to restore tubular mitochondria upon exit from mitosis (Horn et al., 2011). Another cell cycle-dependent kinase, CDK5, is also implicated in phosphorylation at this site in neurons (Strack et al., 2013) (Fig. 1). Under conditions of hypertension and oxidative stress, protein kinase $\mathrm{C} \delta(\mathrm{PKC} \delta)$ phosphorylates

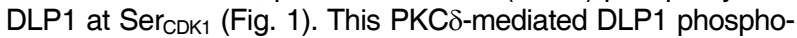
rylation is associated with mitochondrial fragmentation and cell death in oxidative stress conditions (Qi et al., 2011). Ser ${ }_{\mathrm{CDK} 1}$ may also be phosphorylated by the MAP kinase ERK1/2 to increase mitochondrial fission under conditions of high glucoseinduced $\mathrm{Ca}^{2+}$ increase (Yu et al., 2011) (Fig. 1).

\section{ROLE OF THE ER IN REGULATING MITOCHONDRIAL STRUCTURE}

The ER is the largest membranous organelle in the cell. It is the intracellular $\mathrm{Ca}^{2+}$ store and functions in syntheses of secretory proteins, lipids, and other membranous organelles. Early electron micrographs often found the ER and mitochondria in tight apposition. This close physical contact between the ER and mitochondria turned out to be a reflection of the close functional connection of these two organelles, which plays a significant role in cellular physiopathology. The space between the ER and mitochondria at such points of apposition is reported to be 10-25 nm (Csordas et al., 2006). This close association can be biochemically isolated as the mitochondria associated membrane (MAM) fraction, and is functionally implicated in lipid exchange and $\mathrm{Ca}^{2+}$ transfer (Helle et al., 2013; Raturi and Simmen, 2013).

\section{ER-associated mitochondrial fission}

As mentioned earlier, these ER-mitochondria contacts contribute to mitochondrial fission. In both yeast and mammalian cells, ER tubules have been found to wrap around mitochondria, marking sites for fission (Friedman et al., 2011). The ER-associated formin INF2 induces actin assembly at the ER-mitochondria contact, which drives constriction of mitochondrial tubules. This ER-mediated mitochondrial constriction is independent of DLP1 and Mff, and rather it is an initial event providing sites for DLP1 recruitment and assembly for fission (Friedman et al., 2011; Korobova et al., 2013). As for the molecular composition of the ER-mitochondria contact sites participating in mitochondrial fission, a distinct structure in budding yeast called ER mitochondria encounter structure (ERMES) has recently been implicated in mitochondrial fission (Murley et al., 2013).

ERMES is a multi-protein complex found in yeast (Kornmann et al., 2009). The ER protein Maintenance of mitochondrial morphology 1 (Mmm1), the cytosolic protein Mitochondrial distribution and morphology 12 (Mdm12), and mitochondrial outer membrane proteins Mdm10 and Mdm34, are major components of ERMES. Mdm12 and Mdm34 physically tether ER and mitochondria through interaction with Mmm1 and Mdm10 proteins (Burgess et al., 1994; Kornmann et al., 2009). Deletion of any of these four proteins causes failure to form the ERMES complex, and also causes tubular mitochondria to become one or more large spheres, suggesting that ERMES plays a role in controlling mitochondrial morphology (Boldogh et al., 2003; Burgess et al., 1994; Sogo and Yaffe, 1994; Youngman et al., 2004). 


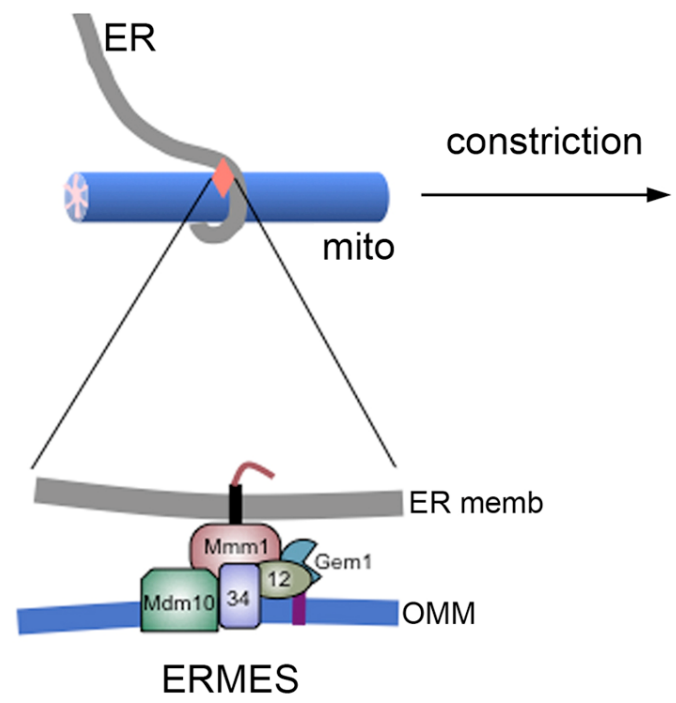

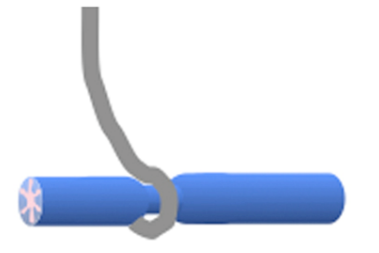
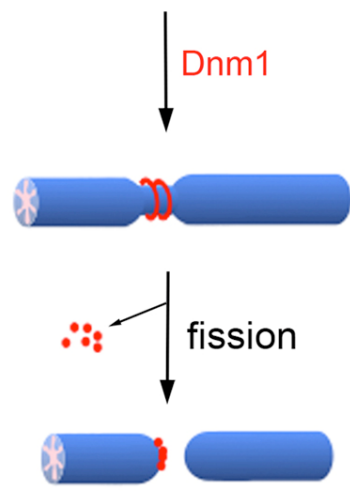

Fig. 2. ER-associated mitochondrial fission in budding yeast. ER-mitochondria contact mediated by ERMES constricts the mitochondrial tubule. The fission protein Dnm1 assembles at the constriction and GTP hydrolysis-mediated contractile force divides mitochondria. OMM, outer mitochondrial membrane.
Moreover, ERMES distributes adjacent to replicating mitochondrial DNA complex (nucleoid), and disruption of ERMES results in disorganized nucleoids and severe mtDNA instability (Hanekamp et al., 2002; Hobbs et al., 2001; Meeusen and Nunnari, 2003). Indeed, ER-associated mitochondrial fission has recently been found coupled with mitochondrial DNA division through ERMES (Murley et al., 2013). In this finding, ERMES foci were localized to sites where mitochondria were constricted and divided. Gem1, a mitochondrial outer membrane-associated Rho-like small GTPase, was found to be associated with this process by releasing mitochondria from their ER contacts, thereby facilitating proper mitochondrial distribution after division. Gem1 is a yeast homologue of the mammalian Miro that functions as a calcium sensor in mitochondrial transport along the cytoskeleton (Cai and Sheng, 2009; Macaskill et al., 2009; Wang and Schwarz, 2009), suggesting the involvement of cytoskeleton in ER-associated mitochondrial fission. In addition, ER-associated mitochondrial fission occurred at sites where newly replicated mitochondrial nucleoids were segregated, which distributed nucleoids to the newly formed mitochondrial tips. These findings provide a new model of mitochondrial fission in which ERMES serves as a spatial link between mitochondrial fission and nucleoid segregation for proper distribution of replicated mitochondrial DNA.

Although ERMES has not been identified in mammals, related observations in mammalian cells suggest functional conservation of a similar structure in coupling of fission and nucleoid segregation. Knockdown of the fission protein DLP1 causes aggregation of nucleoids (Ban-Ishihara et al., 2013). Interestingly, the nucleoid clustering contributes to the formation of tightly stacked cristae, preventing cytochrome $c$ release in apoptotic stimulation (Ban-Ishihara et al., 2013). Miro-1 is also shown to localize at sites where the ER and mitochondria crossover, suggesting that a mechanism similar to ERMESassociated mitochondrial fission also exists in mammalian system (Kornmann et al., 2011).

Interestingly, a recent study suggests that the ER may also be involved in mitochondrial fission independently of DLP1. Infection by Listeria monocytogenes or treatment with pore- forming Listeria toxin listeriolysin (LLO) causes rapid and reversible mitochondria fragmentation (Stavru et al., 2011). The LLO-induced mitochondrial fragmentation occurs at ER crossing sites. Furthermore, inhibition of actin polymerization during the LLO treatment decreases mitochondrial fragmentation, similar to INF2-mediated mitochondrial fission that requires actin assembly (Stavru et al., 2011; 2013). However, upon LLO treatment, DLP1 oligomers are lost from mitochondria and Mff is rapidly degraded, indicating that LLO-mediated mitochondrial fragmentation does not require conventional fission machinery.

\section{Other ER-mitochondria contacts}

The dynamin related fusion protein Mfn2 has been shown to reside in the ER membrane and is tethered to Mfn1 or Mfn2 in the mitochondria, forming an ER-mitochondria contact (de Brito and Scorrano, 2008). This Mfn2-mediated tethering is important for mitochondrial $\mathrm{Ca}^{2+}$ uptake from the ER (de Brito and Scorrano, 2008). However, the aforementioned ER-associated mitochondrial fission does not require Mfn2, suggesting that other types of ER-mitochondria contacts may exist for fission in mammals (Friedman et al., 2011). Interestingly, MARCH5/ MITOL, a mitochondrial outer membrane-associated E3 ubiquitin ligase, interacts with the fission proteins DLP1 and Fis1 (Karbowski et al., 2007; Nakamura et al., 2006; Yonashiro et al., 2006) and activates Mfn2 GTPase activity on mitochondria to increase ER-mitochondria contact (Sugiura et al., 2013). Because MITOL downregulation leads to elongated mitochondria (Karbowski et al., 2007; Sugiura et al., 2013), it is of interest to determine whether the mitochondrial elongation occurs through modifications of fission/fusion factors, through weakened ER-mitochondria contacts for fission, or through a combination of both.

The MAM fraction contains proteins involved in lipid synthesis, ER $\mathrm{Ca}^{2+}$ regulation, and mitochondrial $\mathrm{Ca}^{2+}$ release and uptake (Raturi and Simmen, 2013), suggesting the presence of these proteins at the ER-mitochondria contacts. The chaperon Glucose-Regulated Protein 75 (GRP75) forms a ternary complex with the inositol-1,4,5-triphosphate receptor $\left(\mathrm{IP}_{3} \mathrm{R}\right)$ of the $\mathrm{ER}$, and voltage-dependent anion channel 1 (VDAC1) of the mitochondrial outer membrane (Szabadkai et al., 2006). Inte- 
restingly, the outer membrane protein Mdm10 of the yeast ERMES is structurally related to VDAC, suggesting some similarity in ER-mitochondria contact amongst yeast and higher eukaryotes (Flinner et al., 2013).

In addition, Fis1 at the mitochondrial outer membrane interacts with Bap31, a protein sorting factor at the ER membrane to form a Fis1-Bap31 complex under normal conditions. During Fis1 overexpression-induced apoptosis, procaspase-8 cleaves Bap31 into p20Bap31 in the Fis1-Bap31 complex. Fis1 or Bap31 downregulation decreases the apoptosis induced by exogenous apoptosis inducers such as actinomycin D and etoposide. Caspase-8 activation by the Fis1-Bap31 complex is an early event of apoptosis and is found to be independent of mitochondrial fission (Iwasawa et al., 2011). It remains unclear whether these above-mentioned interactions are components of one physical subunit complex such as ERMES, or whether they exist in separate functional complexes.

\section{CONCLUSION}

This review has touched upon mechanisms and regulation of mitochondrial fission. Although we limited our discussion to the phosphorylation-mediated regulation of mitochondrial fission, many other mechanisms have been reported to regulate not only mitochondrial fission but also fusion. Because fission and fusion must be balanced to maintain proper mitochondrial morphology and distribution, the regulatory mechanisms of mitochondrial fission and fusion are predicted to intersect at some points in the signaling cascade.

With a view to the bigger picture, autophagic removal of mitochondria (mitophagy), although it is not discussed here, is known to be closely connected to mitochondrial dynamics. In considering mitochondrial homeostasis, the connection between mitochondrial dynamics and biogenesis cannot be ignored. Therefore, mitochondrial biogenesis, dynamics, and mitophagy would constitute the components of mitochondrial homeostasis in order to properly maintain mitochondrial function, potentially through common regulatory mechanisms. A recent development elucidating the role of the ER in mitochondrial fission has provided new insights as to structural and functional communication between the two organelles. While the detailed mechanisms of ER-associated mitochondrial fission need to be investigated further, the already-recognized role of the ERmitochondrial contact (MAM) in lipid and $\mathrm{Ca}^{2+}$ transfer may be an important factor in mitochondrial fission as well. Although the role of the MAM in ER-mediated mitochondrial fission is not yet established, the specific lipid environment formed on the mitochondrial surface through ER-mitochondria lipid exchange may attract necessary fission proteins. In addition, $\mathrm{Ca}^{2+}$ is a wellknown regulator of both mitochondrial function and dynamics. Given the gaining traction of current research on ER-mitochondrial contacts, a considerable advance in this aspect of mitochondrial dynamics can be expected in near future.

\section{ACKNOWLEDGMENTS}

This work was supported by National Institutes of Health Grants DK078618 and DK061991 to YY.

\section{REFERENCES}

Ban, T., Heymann, J.A., Song, Z., Hinshaw, J.E., and Chan, D.C. (2010). OPA1 disease alleles causing dominant optic atrophy have defects in cardiolipin-stimulated GTP hydrolysis and membrane tubulation. Hum. Mol. Genet. 19, 2113-2122.

Ban-Ishihara, R., Ishihara, T., Sasaki, N., Mihara, K., and Ishihara, N. (2013). Dynamics of nucleoid structure regulated by mito- chondrial fission contributes to cristae reformation and release of cytochrome c. Proc. Natl. Acad. Sci. USA 110, 11863-11868.

Boldogh, I.R., Nowakowski, D.W., Yang, H.C., Chung, H., Karmon, S., Royes, P., and Pon, L.A. (2003). A protein complex containing Mdm10p, Mdm12p, and Mmm1p links mitochondrial membranes and DNA to the cytoskeleton-based segregation machinery. Mol. Biol. Cell 14, 4618-4627.

Burgess, S.M., Delannoy, M., and Jensen, R.E. (1994). MMM1 encodes a mitochondrial outer membrane protein essential for establishing and maintaining the structure of yeast mitochondria. J. Cell Biol. 126, 1375-1391.

Cai, Q., and Sheng, Z.H. (2009). Moving or stopping mitochondria: Miro as a traffic cop by sensing calcium. Neuron 61, 493-496.

Cereghetti, G.M., Stangherlin, A., Martins de Brito, O., Chang, C.R., Blackstone, C., Bernardi, P., and Scorrano, L. (2008). Dephosphorylation by calcineurin regulates translocation of Drp1 to mitochondria. Proc. Natl. Acad. Sci. USA 105, 15803-15808.

Chang, C.R., and Blackstone, C. (2007). Cyclic AMP-dependent protein kinase phosphorylation of Drp1 regulates its GTPase activity and mitochondrial morphology. J. Biol. Chem. 282, 2158321587.

Cho, D.H., Nakamura, T., Fang, J., Cieplak, P., Godzik, A., Gu, Z., and Lipton, S.A. (2009). S-nitrosylation of Drp1 mediates betaamyloid-related mitochondrial fission and neuronal injury. Science 324, 102-105.

Cribbs, J.T., and Strack, S. (2007). Reversible phosphorylation of Drp1 by cyclic AMP-dependent protein kinase and calcineurin regulates mitochondrial fission and cell death. EMBO Rep. 8, 939-944.

Csordas, G., Renken, C., Varnai, P., Walter, L., Weaver, D., Buttle, K.F., Balla, T., Mannella, C.A., and Hajnoczky, G. (2006). Structural and functional features and significance of the physical linkage between ER and mitochondria. J. Cell Biol. 174, 915-921.

de Brito, O.M., and Scorrano, L. (2008). Mitofusin 2 tethers endoplasmic reticulum to mitochondria. Nature $456,605-610$.

Dickey, A.S., and Strack, S. (2011). PKA/AKAP1 and PP2A/B $\beta 2$ regulate neuronal morphogenesis via Drp1 phosphorylation and mitochondrial bioenergetics. J. Neurosci. 31, 15716-15726.

Flinner, N., Ellenrieder, L., Stiller, S.B., Becker, T., Schleiff, E., and Mirus, O. (2013). Mdm10 is an ancient eukaryotic porin co-occurring with the ERMES complex. Biochim. Biophys. Acta 1833, 3314-3325.

Friedman, J.R., Lackner, L.L., West, M., DiBenedetto, J.R., Nunnari, J., and Voeltz, G.K. (2011). ER tubules mark sites of mitochondrial division. Science 334, 358-362.

Gawlowski, T., Suarez, J., Scott, B., Torres-Gonzalez, M., Wang, H. Schwappacher, R., Han, X., Yates, J.R., 3rd, Hoshijima, M., and Dillmann, W. (2012). Modulation of dynamin-related protein 1 (DRP1) function by increased O-linked-beta-N-acetylglucosamine modification (O-GlcNAc) in cardiac myocytes. J. Biol. Chem. 287, 30024-30034.

Gomes, L.C., Di Benedetto, G., and Scorrano, L. (2011). During autophagy mitochondria elongate, are spared from degradation and sustain cell viability. Nat. Cell Biol. 13, 589-598

Griffin, E.E., Graumann, J., and Chan, D.C. (2005). The WD40 protein Caf $4 p$ is a component of the mitochondrial fission machinery and recruits Dnm1p to mitochondria. J. Cell Biol. 170, 237-248.

Guo, C., Hildick, K.L., Luo, J., Dearden, L., Wilkinson, K.A., and Henley, J.M. (2013). SENP3-mediated deSUMOylation of dynaminrelated protein 1 promotes cell death following ischaemia. EMBO J. 32, 1514-1528.

Han, X.J., Lu, Y.F., Li, S.A., Kaitsuka, T., Sato, Y., Tomizawa, K., Nairn, A.C., Takei, K., Matsui, H., and Matsushita, M. (2008). CaM kinase l $\alpha$-induced phosphorylation of Drp1 regulates mitochondrial morphology. J. Cell Biol. 182, 573-585.

Hanekamp, T., Thorsness, M.K., Rebbapragada, I., Fisher, E.M., Seebart, C., Darland, M.R., Coxbill, J.A., Updike, D.L., and Thorsness, P.E. (2002). Maintenance of mitochondrial morphology is linked to maintenance of the mitochondrial genome in Saccharomyces cerevisiae. Genetics 162, 1147-1156.

Helle, S.C., Kanfer, G., Kolar, K., Lang, A., Michel, A.H., and Kornmann, B. (2013). Organization and function of membrane contact sites. Biochim. Biophys. Acta 1833, 2526-2541.

Hinshaw, J.E. (2000). Dynamin and its role in membrane fission. Annu. Rev. Cell Dev. Biol. 16, 483-519.

Hobbs, A.E., Srinivasan, M., McCaffery, J.M., and Jensen, R.E. (2001). 
Mmm1p, a mitochondrial outer membrane protein, is connected to mitochondrial DNA (mtDNA) nucleoids and required for mtDNA stability. J. Cell Biol. 152, 401-410.

Horn, S.R., Thomenius, M.J., Johnson, E.S., Freel, C.D., Wu, J.Q., Coloff, J.L., Yang, C.S., Tang, W., An, J., Ilkayeva, O.R., et al. (2011). Regulation of mitochondrial morphology by APC/CCdh1mediated control of Drp1 stability. Mol. Biol. Cell 22, 1207-1216.

Iwasawa, R., Mahul-Mellier, A.L., Datler, C., Pazarentzos, E., and Grimm, S. (2011). Fis1 and Bap31 bridge the mitochondria-ER interface to establish a platform for apoptosis induction. EMBO J. 30, 556-568.

Karbowski, M., Neutzner, A., and Youle, R.J. (2007). The mitochondrial E3 ubiquitin ligase MARCH5 is required for Drp1 dependent mitochondrial division. J. Cell Biol. 178, 71-84

Kashatus, D.F., Lim, K.H., Brady, D.C., Pershing, N.L., Cox, A.D., and Counter, C.M. (2011). RALA and RALBP1 regulate mitochondrial fission at mitosis. Nat. Cell Biol. 13, 1108-1115.

Kim, H., Scimia, M.C., Wilkinson, D., Trelles, R.D., Wood, M.R., Bowtell, D., Dillin, A., Mercola, M., and Ronai, Z.A. (2011). Finetuning of Drp1/Fis1 availability by AKAP121/Siah2 regulates mitochondrial adaptation to hypoxia. Mol. Cell 44, 532-544.

Kornmann, B., Currie, E., Collins, S.R., Schuldiner, M., Nunnari, J., Weissman, J.S., and Walter, P. (2009). An ER-mitochondria tethering complex revealed by a synthetic biology screen. Science 325, 477-481.

Kornmann, B., Osman, C., and Walter, P. (2011). The conserved GTPase Gem1 regulates endoplasmic reticulum-mitochondria connections. Proc. Natl. Acad. Sci. USA 108, 14151-14156.

Korobova, F., Ramabhadran, V., and Higgs, H.N. (2013). An actindependent step in mitochondrial fission mediated by the ERassociated formin INF2. Science 339, 464-467.

Loson, O.C., Song, Z., Chen, H., and Chan, D.C. (2013). Fis1, Mff, MiD49, and MiD51 mediate Drp1 recruitment in mitochondrial fission. Mol. Biol. Cell 24, 659-667.

Macaskill, A.F., Rinholm, J.E., Twelvetrees, A.E., Arancibia-Carcamo, I.L., Muir, J., Fransson, A., Aspenstrom, P., Attwell, D., and Kittler, J.T. (2009). Miro1 is a calcium sensor for glutamate receptor-dependent localization of mitochondria at synapses. Neuron 61, 541-555.

McNiven, M.A., Cao, H., Pitts, K.R., and Yoon, Y. (2000). The dynamin family of mechanoenzymes: pinching in new places. Trends Biochem. Sci. 25, 115-120.

Mears, J.A., Lackner, L.L., Fang, S., Ingerman, E., Nunnari, J., and Hinshaw, J.E. (2011). Conformational changes in Dnm1 support a contractile mechanism for mitochondrial fission. Nat. Struct. Mol. Biol. 18, 20-26.

Meeusen, S., and Nunnari, J. (2003). Evidence for a two membranespanning autonomous mitochondrial DNA replisome. J. Cell Biol. 163, 503-510.

Merrill, R.A., Dagda, R.K., Dickey, A.S., Cribbs, J.T., Green, S.H., Usachev, Y.M., and Strack, S. (2011). Mechanism of neuroprotective mitochondrial remodeling by PKA/AKAP1. PLoS Biol. 9, e1000612.

Murley, A., Lackner, L.L., Osman, C., West, M., Voeltz, G.K., Walter, P., and Nunnari, J. (2013). ER-associated mitochondrial division links the distribution of mitochondria and mitochondrial DNA in yeast. eLife 2, e00422.

Nakamura, N., Kimura, Y., Tokuda, M., Honda, S., and Hirose, S. (2006). MARCH-V is a novel mitofusin 2- and Drp1-binding protein able to change mitochondrial morphology. EMBO Rep. 7, 1019-1022.

Otera, H., Wang, C., Cleland, M.M., Setoguchi, K., Yokota, S., Youle, R.J., and Mihara, K. (2010). Mff is an essential factor for mitochondrial recruitment of Drp1 during mitochondrial fission in mammalian cells. J. Cell Biol. 191, 1141-1158.

Palmer, C.S., Osellame, L.D., Laine, D., Koutsopoulos, O.S., Frazier, A.E., and Ryan, M.T. (2011). MiD49 and MiD51, new components of the mitochondrial fission machinery. EMBO Rep. 12, 565-573.

Palmer, C.S., Elgass, K.D., Parton, R.G., Osellame, L.D., Stojanovski, D., and Ryan, M.T. (2013). Adaptor proteins MiD49 and MiD51 can act independently of Mff and Fis1 in Drp1 recruitment and are specific for mitochondrial fission. J. Biol. Chem. 288, 27584-27593.

Qi, X., Disatnik, M.H., Shen, N., Sobel, R.A., and Mochly-Rosen, D. (2011). Aberrant mitochondrial fission in neurons induced by protein kinase $\mathrm{C} \delta$ under oxidative stress conditions in vivo. Mol. Biol. Cell 22, 256-265.

Raturi, A., and Simmen, T. (2013). Where the endoplasmic reticulum and the mitochondrion tie the knot: the mitochondria-associated membrane (MAM). Biochim. Biophys. Acta 1833, 213-224.

Slupe, A.M., Merrill, R.A., Flippo, K.H., Lobas, M.A., Houtman, J.C. and Strack, S. (2013). A calcineurin docking motif (LXVP) in dynamin-related protein 1 contributes to mitochondrial fragmentation and ischemic neuronal injury. J. Biol. Chem. 288, 12353-12365.

Smirnova, E., Griparic, L., Shurland, D.L., and van der Bliek, A.M. (2001). Dynamin-related protein Drp1 is required for mitochondrial division in mammalian cells. Mol. Biol. Cell 12, 2245-2256.

Sogo, L.F., and Yaffe, M.P. (1994). Regulation of mitochondrial morphology and inheritance by Mdm10p, a protein of the mitochondrial outer membrane. J. Cell Biol. 126, 1361-1373.

Strack, S., Wilson, T.J., and Cribbs, J.T. (2013). Cyclin-dependent kinases regulate splice-specific targeting of dynamin-related protein 1 to microtubules. J. Cell Biol. 201, 1037-1051.

Stavru, F., Bouillaud, F., Sartori, A., Ricquier, D., and Cossart, P. (2011). Listeria monocytogenes transiently alters mitochondrial dynamics during infection. Proc. Natl. Acad. Sci. USA 108, 3612- 3617.

Stavru, F., Palmer, A.E., Wang, C., Youle, R.J., and Cossart, P. (2013). Atypical mitochondrial fission upon bacterial infection. Proc. Natl. Acad. Sci. USA 110, 16003-16008.

Sugiura, A., Nagashima, S., Tokuyama, T., Amo, T., Matsuki, Y., Ishido, S., Kudo, Y., McBride, H.M., Fukuda, T., Matsushita, N., et al. (2013). MITOL regulates endoplasmic reticulum-mitochondria contacts via Mitofusin2. Mol. Cell 51, 20-34.

Szabadkai, G., Bianchi, K., Varnai, P., De Stefani, D., Wieckowski, M.R., Cavagna, D., Nagy, A.I., Balla, T., and Rizzuto, R. (2006) Chaperone-mediated coupling of endoplasmic reticulum and mitochondrial $\mathrm{Ca}^{2+}$ channels. J. Cell Biol. 175, 901-911.

Taguchi, N., Ishihara, N., Jofuku, A., Oka, T., and Mihara, K. (2007). Mitotic phosphorylation of dynamin-related GTPase Drp1 participates in mitochondrial fission. J. Biol. Chem. 282, 11521-11529.

Tieu, Q., Okreglak, V., Naylor, K., and Nunnari, J. (2002). The WD repeat protein, Mdv1p, functions as a molecular adaptor by interacting with Dnm1p and Fis1p during mitochondrial fission. J. Cell Biol. 158, 445-452.

Wang, X., and Schwarz, T.L. (2009). The mechanism of $\mathrm{Ca}^{2+}$-dependent regulation of kinesin-mediated mitochondrial motility. Cell 136, 163-174.

Wang, J.X., Jiao, J.Q., Li, Q., Long, B., Wang, K., Liu, J.P., Li, Y.R., and Li, P.F. (2011). miR-499 regulates mitochondrial dynamics by targeting calcineurin and dynamin-related protein-1. Nat. Med. 17, 71-78.

Wang, W., Wang, Y., Long, J., Wang, J., Haudek, S.B., Overbeek, P., Chang, B.H., Schumacker, P.T., and Danesh, F.R. (2012a). Mitochondrial fission triggered by hyperglycemia is mediated by ROCK1 activation in podocytes and endothelial cells. Cell Metabol. 15, 186-200.

Wang, Z., Jiang, H., Chen, S., Du, F., and Wang, X. (2012b). The mitochondrial phosphatase PGAM5 functions at the convergence point of multiple necrotic death pathways. Cell 148, 228-243.

Wasiak, S., Zunino, R., and McBride, H.M. (2007). Bax/Bak promote sumoylation of DRP1 and its stable association with mitochondria during apoptotic cell death. J. Cell Biol. 177, 439-450.

Yonashiro, R., Ishido, S., Kyo, S., Fukuda, T., Goto, E., Matsuki, Y., Ohmura-Hoshino, M., Sada, K., Hotta, H., Yamamura, H., et al. (2006). A novel mitochondrial ubiquitin ligase plays a critical role in mitochondrial dynamics. EMBO J. 25, 3618-3626.

Yoon, Y., Pitts, K.R., and McNiven, M.A. (2001). Mammalian dynamin-like protein DLP1 tubulates membranes. Mol. Biol. Cell 12, 2894-2905

Youngman, M.J., Hobbs, A.E., Burgess, S.M., Srinivasan, M., and Jensen, R.E. (2004). Mmm2p, a mitochondrial outer membrane protein required for yeast mitochondrial shape and maintenance of mtDNA nucleoids. J. Cell Biol. 164, 677-688.

Yu, T., Jhun, B.S., and Yoon, Y. (2011). High-glucose stimulation increases reactive oxygen species production through the calcium and mitogen-activated protein kinase-mediated activation of mitochondrial fission. Antioxid. Redox Signal. 14, 425-437.

Zhao, J., Liu, T., Jin, S., Wang, X., Qu, M., Uhlen, P., Tomilin, N., Shupliakov, O., Lendahl, U., and Nister, M. (2011). Human MIEF1 recruits Drp1 to mitochondrial outer membranes and promotes mitochondrial fusion rather than fission. EMBO J. 30, 2762-2778. 